\title{
Open versus minimally invasive total gastrectomy after neoadjuvant chemotherapy: results of a European randomized trial
}

\author{
Nicole van der Wielen ${ }^{1}$ (1) . Jennifer Straatman ${ }^{1,2} \cdot$ Freek Daams $^{1} \cdot$ Riccardo Rosati $^{3} \cdot$ Paolo Parise $^{3}$. \\ Jürgen Weitz ${ }^{4}$. Christoph Reissfelder ${ }^{5} \cdot$ Ismael Diez del Val $^{6}$. Carlos Loureiro ${ }^{6}$ Purificación Parada-González ${ }^{7}$. \\ Elena Pintos-Martínez ${ }^{7}$. Francisco Mateo Vallejo ${ }^{8}$. Carlos Medina Achirica ${ }^{8}$. Andrés Sánchez-Pernaute ${ }^{9}$. \\ Adriana Ruano Campos $^{9}$ - Luigi Bonavina ${ }^{10}$. Emanuele L. G. Asti ${ }^{10}$ - Alfredo Alonso Poza ${ }^{11}$. Carlos Gilsanz ${ }^{11}$. \\ Magnus Nilsson $^{12}$. Mats Lindblad ${ }^{12}$. Suzanne S. Gisbertz ${ }^{13} \cdot$ Mark I. van Berge Henegouwen $^{13}$. \\ Uberto Fumagalli Romario ${ }^{14}$. Stefano De Pascale ${ }^{14} \cdot$ Khurshid Akhtar $^{15} \cdot$ H. Jaap Bonjer ${ }^{1} \cdot$ Miguel A. Cuesta $^{1}$. \\ Donald L. van der Peet ${ }^{1}$
}

Received: 2 April 2020 / Accepted: 17 July 2020 / Published online: 31 July 2020

(c) The Author(s) 2020

\begin{abstract}
Background Surgical resection with adequate lymphadenectomy is regarded the only curative option for gastric cancer. Regarding minimally invasive techniques, mainly Asian studies showed comparable oncological and short-term postoperative outcomes. The incidence of gastric cancer is lower in the Western population and patients often present with more advanced stages of disease. Therefore, the reproducibility of these Asian results in the Western population remains to be investigated. Methods A randomized trial was performed in thirteen hospitals in Europe. Patients with an indication for total gastrectomy who received neoadjuvant chemotherapy were eligible for inclusion and randomized between open total gastrectomy (OTG) or minimally invasive total gastrectomy (MITG). Primary outcome was oncological safety, measured as the number of resected lymph nodes and radicality. Secondary outcomes were postoperative complications, recovery and 1-year survival. Results Between January 2015 and June 2018, 96 patients were included in this trial. Forty-nine patients were randomized to OTG and 47 to MITG. The mean number of resected lymph nodes was $43.4 \pm 17.3$ in OTG and $41.7 \pm 16.1$ in MITG $(p=0.612)$. Forty-eight patients in the OTG group had a R0 resection and 44 patients in the MITG group $(p=0.617)$. Oneyear survival was $90.4 \%$ in OTG and $85.5 \%$ in MITG $(p=0.701)$. No significant differences were found regarding postoperative complications and recovery.

Conclusion These findings provide evidence that MITG after neoadjuvant therapy is not inferior regarding oncological quality of resection in comparison to OTG in Western patients with resectable gastric cancer. In addition, no differences in postoperative complications and recovery were seen.
\end{abstract}

Keywords Minimally invasive total gastrectomy $\cdot$ Neoadjuvant chemotherapy

\section{Introduction}

Gastric cancer remains one of the most common cancers worldwide, but its incidence varies widely. A high incidence is observed in East-Asia with an incidence of up to 60 patients per 100.000 inhabitants in Korea, whereas the incidence in Northwestern Europe and North America is low, with an incidence of around 5.8 per 100.000 [1]. Based

Nicole van der Wielen

ni.vanderwielen@amsterdamumc.nl

Extended author information available on the last page of the article on the results of the MAGIC trail and in accordance with the consensus guidelines of the European Society for Medical Oncology (ESMO), radical gastrectomy with free margins and an adequate lymphadenectomy, and if indicated along with perioperative neoadjuvant chemotherapy, is the standard of care in patients with advanced gastric cancer [2, 3].

With the introduction of minimally invasive techniques in the treatment of gastric cancer, several large Asian studies show beneficial short-term outcomes and comparable long-term outcomes in the treatment of gastric cancer [4-6]. It should be noted that these results are obtained in centers with a high volume of gastric cancer patients in earlier stages 
of disease due to the availability of screening programs [7, 8]. Not only is the incidence of gastric cancer lower in the West, but patients present themselves with more advanced stages of disease, often localized more proximally in the stomach $[1,9]$. Most studies regarding minimally invasive techniques in the treatment of gastric cancer have focused on partial gastrectomy. Minimally invasive total gastrectomy (MITG) remains a challenge due to oncological and technical aspects.

The potential advantages; such as reduced operative trauma may lead to reduced postoperative complications and improved postoperative recovery whilst preserving oncological outcomes. These potential benefits have led to widespread adoption of minimally invasive gastrectomy. The results of the MAGIC trial showed that perioperative chemotherapy reduced tumor size and increased progression-free and overall survival in gastric cancer patients. The results of perioperative FLOT versus perioperative ECF in advanced gastric cancer showed a better overall survival in the FLOT group. Based on these results neoadjuvant chemotherapy is standard of care in the treatment of advanced gastric cancer in the West, as advised by the European guidelines [10, 11]. To date no randomized clinical trial has investigated the benefits of MITG, following neoadjuvant therapy, in the Western population. One trial assessed open total gastrectomy (OTG) and MITG, before the implementation of neoadjuvant therapy [12].

We hypothesize that a novel surgical technique such as MITG should at least be non-inferior with regard to oncological quality of the resection, with similar or improved postoperative outcomes and survival. Oncological quality of the resection was measured as the number of retrieved lymph nodes in the corresponding stations and radicality (negative circumferential resection margin) of the procedure.

\section{Methods}

\section{Study design}

A non-inferiority, multicenter, international, randomized trial between January 2015 and June 2018 was performed in 13 hospitals in six European countries. Hospitals in the Netherlands, Sweden, Spain, Italy, Germany and the United Kingdom participated in this trial. The medical ethics board of all participating hospitals approved the trial protocol prior to inclusion of patients.

Eligible patients had resectable adenocarcinoma of the stomach and were treated with neoadjuvant chemotherapy, and based on location of the tumor, total gastrectomy was indicated. Patients had to be above 18 years of age and an American Society of Anesthesiologist classification of 3 or lower. Patients with early gastric cancer suitable for local treatment, distal gastric cancer suitable for distal gastrectomy, gastro-esophageal junction tumors, previous surgery of the stomach and previous or co-existing other malignancies were excluded [13].

For quality assurance, the principal investigator visited all centers interested in trial participation for a site initiation. With a low incidence of gastric cancer in the West, all gastrectomies are performed in specialized hospitals to maintain quality of care for these patients. To prevent surgeon bias, both procedures were done by surgeons experienced in open and minimally invasive gastrectomy. Surgeons eligible for participation had to have experience with at least 20 laparoscopic gastrectomies, consisting of at least ten total gastrectomies. Because this study reflected current practice, no additional quality measures were deemed necessary.

Diagnosis and staging were established before neoadjuvant chemotherapy by esophagogastroscopy, with biopsies and CT scan. Additional diagnostics such as PET-CT, MRI, ultrasound or diagnostic laparoscopy could be performed if deemed necessary. A diagnostic laparoscopy before the start of neoadjuvant chemotherapy was advised if there was doubt regarding resectability. Surgeons at the outpatient clinic informed eligible patients of the treatment regimen. Written informed consent was obtained from included patients.

\section{Randomization}

A computer-generated randomization tool was used to randomize the patients in a 1:1 ratio to either an open or a minimally invasive procedure. Randomization was stratified per study center and performed in blocks of six. Randomization took place after assessment of the inclusion and exclusion criteria and after signing the informed consent form. Both patient and the surgeon were not blinded for the allocated procedure. In one hospital a coin flip on the operating room was used for randomization of several patients due to an error with the online randomization tool at that time. These patients were added as soon as the tool was working again, to ensure block randomization was still correct. To assure the quality of the primary outcome regarding lymphadenectomy and radicality, the pathologist was blinded for the type of surgery.

\section{Procedures}

All patients received neoadjuvant chemotherapy in accordance with local protocols. An overview of types of neoadjuvant chemotherapy are depicted in Table 1.

Anaesthesia was similar for patients in open or minimally invasive surgery. All patients received a single, prophylactic gift of antibiotics according to the local protocol in the participating centers 30 min preoperatively. 


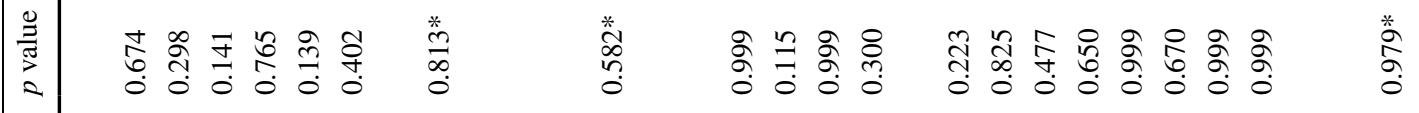

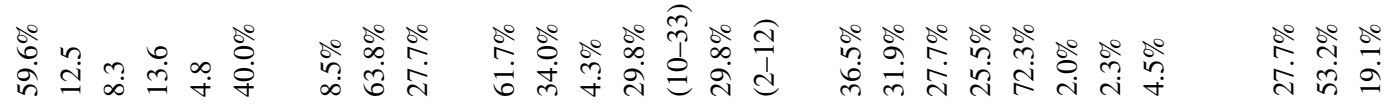

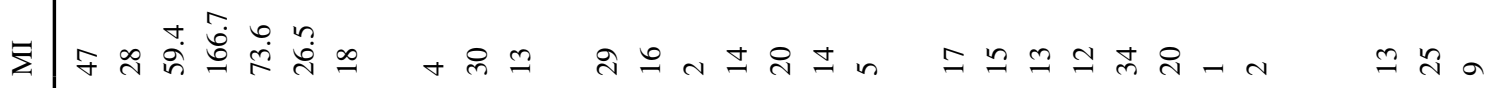

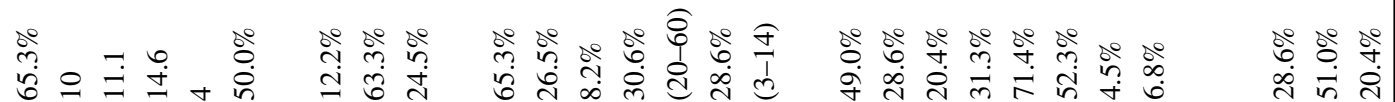 \\ 苛}

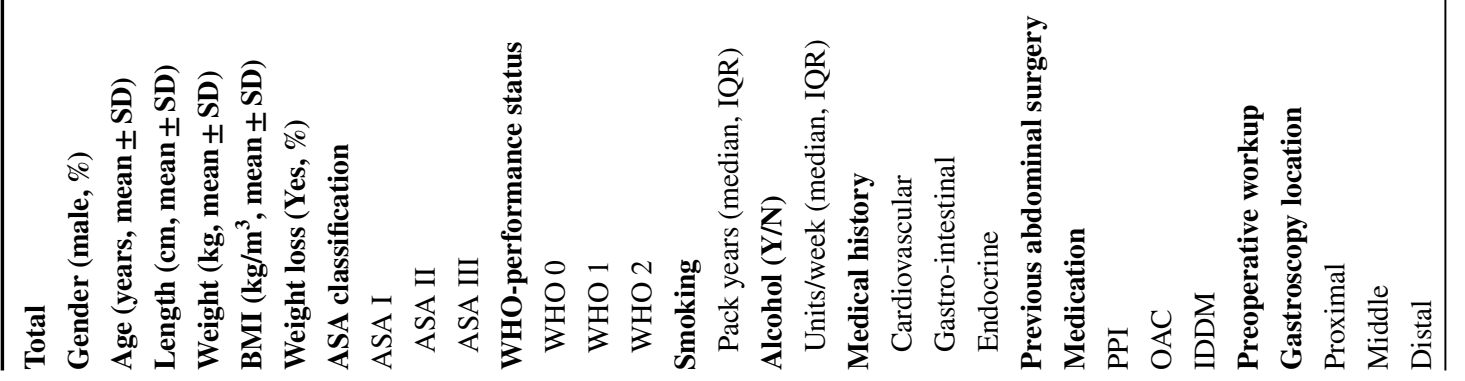




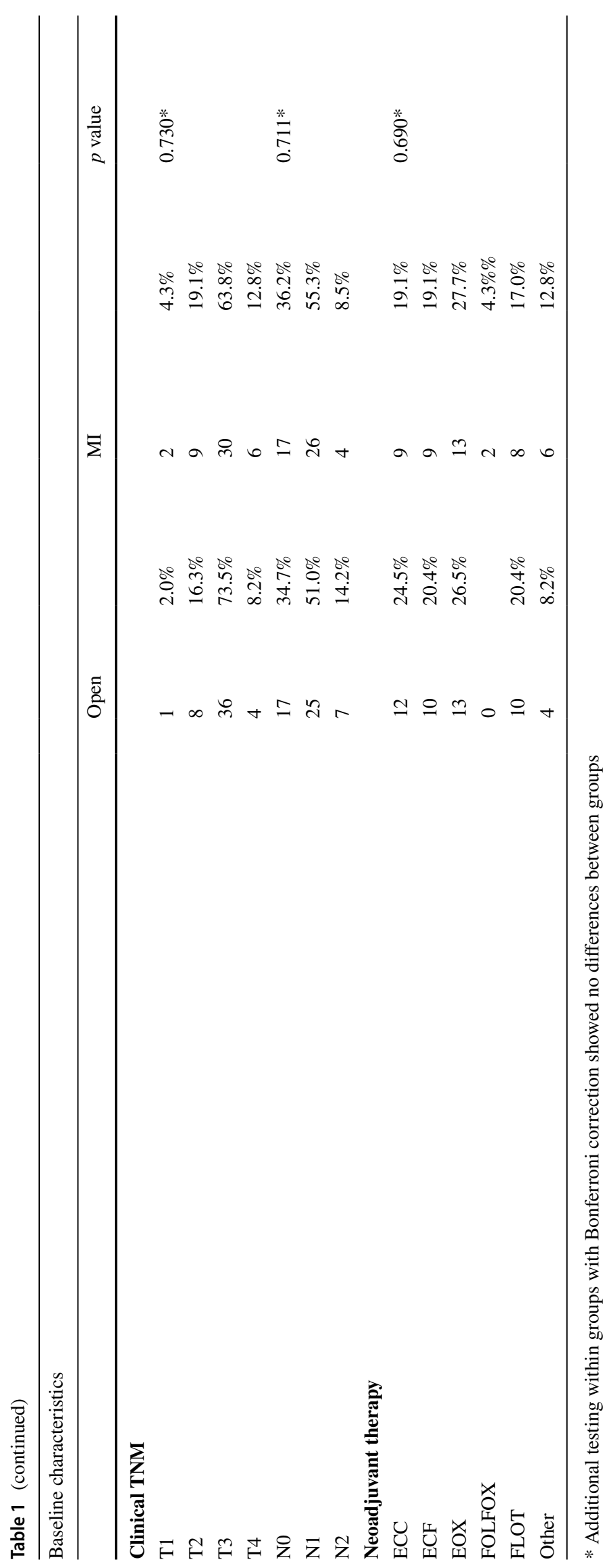


Gastrectomy was performed 6-8 weeks after completion of neoadjuvant chemotherapy. Access to the abdomen in the OTG group was obtained via a median laparotomy. In the MITG group, the placement and number of trocars were based on surgeon preferences. An overview of number of trocars and site of extraction are depicted in Table 2.

Recommended lymph node dissection consisted of a D2 lymphadenectomy, being lymph node stations $1-7,8 \mathrm{a}, 9$, $10,11 \mathrm{p}$ and $12 \mathrm{a}$ [14]. After extraction of the specimen the surgeon attached numbered tags in correspondence with the number of the lymph node station. The specimen was analysed by the pathologist for radicality, the number of resected lymph nodes with the corresponding lymph node stations and response to neoadjuvant chemotherapy according to Mandard [15]. The pathologists had separate access to the online case report forms to enter their results.

Postoperative treatment was similar in both groups. A nasojejunal tube could be inserted according to local preference. Enteral feeding could be resumed depending on the clinical status of the patient. Patients were encouraged to get out of bed and start walking with the help of a physical therapist or nurse. Follow-up took place 3, 6 and 12 months postoperatively, or sooner if deemed necessary.

\section{Study outcomes}

The number of resected lymph nodes with a D2 lymphadenectomy according to the definition of the Japanese Gastric Cancer Association, alongside radicality, is a marker for the quality of care and, therefore, our primary outcome [14]. A standardized report regarding tumor size, radicality, number of resected lymph nodes, resected lymph node stations and Mandard scores was conducted [15].

Secondary outcomes regarded operative results such as operation duration, blood loss and perioperative complications. Postoperative outcomes regarded morbidity and mortality with postoperative mortality being defined as 30-day mortality or in hospital mortality. Complications were graded according to the Clavien-Dindo classification and all complications per patient were assessed using the comprehensive complication index $[16,17]$. Complications requiring invasive intervention of admission to an intensive care unit, as measured by a Clavien-Dindo score of $3 \mathrm{~A}$ or higher, were deemed major complications.

In addition, postoperative recovery such as time to first intake, time to first flatus and hospital stay was measured. In the overall analysis, textbook outcome was determined
Table 2 Operative details

\begin{tabular}{|c|c|c|c|c|c|}
\hline \multicolumn{6}{|l|}{ Operative details } \\
\hline & Open $(n=49)$ & & MI $(n=47)$ & & $p$-value \\
\hline $\begin{array}{l}\text { Duration of surgery (median, } \\
\text { IQR) }\end{array}$ & 200 & $(164-245)$ & 244 & $(198-293)$ & 0.005 \\
\hline Blood loss, ml (median, IQR) & 200 & $(100-400)$ & 171 & $(64-300)$ & 0.454 \\
\hline Transfusion $(\mathrm{Y} / \mathrm{N})$ & 4 & $8.2 \%$ & 2 & $4.3 \%$ & 0.678 \\
\hline \multicolumn{6}{|l|}{ Peri-operative complications } \\
\hline Bleeding & 4 & $8.3 \%$ & 5 & $10.6 \%$ & $0.774 *$ \\
\hline Bowel perforation & 1 & $2.1 \%$ & 0 & & \\
\hline Arrythmia & 1 & $2.1 \%$ & 1 & $2.1 \%$ & \\
\hline Drain & 39 & $83.0 \%$ & 33 & $70.2 \%$ & 0.223 \\
\hline SMEQ-score (median, IQR) & 12.5 & $(0-67)$ & 50 & $(0-78.5)$ & 0.393 \\
\hline \multicolumn{6}{|c|}{ Minimally invasive total gastrectomy } \\
\hline \multicolumn{6}{|c|}{ Number of trocars } \\
\hline 3 trocars & & & 5 & $10.6 \%$ & \\
\hline 4 trocars & & & 12 & $25.5 \%$ & \\
\hline 5 trocars & & & 27 & $57.4 \%$ & \\
\hline 6 trocars & & & 3 & $6.4 \%$ & \\
\hline \multicolumn{6}{|l|}{ Extraction site } \\
\hline Pfannenstiehl & & & 23 & $48.9 \%$ & \\
\hline Epigastrio & & & 4 & $8.5 \%$ & \\
\hline Left pararectal & & & 9 & $19.1 \%$ & \\
\hline Midline mini laparotomy & & & 11 & $23.4 \%$ & \\
\hline Conversion & & & 3 & $6.4 \%$ & \\
\hline \multicolumn{6}{|l|}{ Open total gastrectomy } \\
\hline Length of Incision, cm & 20 & $(17-32)$ & & & \\
\hline
\end{tabular}

* Additional testing within groups with Bonferroni correction showed no differences between groups 
for each patient. A textbook outcome was achieved if: a curative resection was performed, with no perioperative complications, a pathological radical resection (R0) with more than 15 lymph nodes resected, no major complications (Clavien-Dindo $3 \mathrm{~A}$ or higher), no reinterventions, a length of hospital stay less than 21 days, no mortality in the first 30 days and no readmission in the first 30 days [18].

Data regarding 1-year disease free and overall survival was collected.

\section{Statistical analysis}

The sample size was calculated based on two previous studies. The first sample size was based on the Dutch cancer registry of 2013, with a median yield of 19 lymph nodes, for non-inferiority with a power of $90 \%$ and one-sided $t$ test with a significance level $(\alpha)$ of 0.05 and a non-inferiority margin of -4 , a total of 66 patients per group were deemed necessary $[19,20]$.

The second sample size was based on a large western study regarding patients that underwent gastrectomy for cancer with curative intent and described lymph node yield following neoadjuvant therapy in a Western population [21]. For a non-inferiority test with $90 \%$ power, and one-sided $t$ test with a significance level $(\alpha)$ of 0.05 , a standard deviation of 7.8 and non-inferiority margin of -5 a total of 48 patients per group were to be included. Although the second sample size was more in fit with the study, we were confident that we could reach our first sample size. Accrual started according to the expected scheme, but accrual was slow. Based on the advice of the statistician and medical ethical review board, the research group decided to reassess the sample size.

The main reason for this slow accrual was a large group of patients that did not meet the inclusion criteria, mainly patients were not deemed fit enough for neoadjuvant chemotherapy.

Statistical analysis was performed using SPSS statistical package, version 22 (IBM software). Open and minimally invasive total gastrectomy were compared with continuous variables described as means and standard deviation for normal distributions and medians and interquartile ranges for non-normal distributions. Analysis was performed as intention to treat. Comparison tests were performed with Student's $t$ test and Mann-Whitney $U$ tests as appropriate.

Frequencies were described as number and percentage of total. Comparison was performed with Chi square tests, for variables with multiple categories additional testing within groups was performed with Bonferroni correction. A twosided $p$ value of 0.05 was deemed statistically significant. Correction for possible confounders or effect modifiers was assessed using logistic regression techniques. Kaplan-Meier curves with a Mantel-Cox log rank test were used for survival. Cox regression was used to analyze confounders and effect-modifiers.

\section{Results}

\section{Patient characteristics}

A total of 110 patients of 134 eligible patients were included in this trial and randomly assigned to undergo an open or minimally invasive gastrectomy. In ten patients, gastrectomy was not performed due to peritoneal carcinomatosis at the time of surgery. These patients were excluded from further analyses. Four patients retracted from the trial and therefor the data could not be used for analyses, thus resulting in 96 patients. A flow chart of the included patients is depicted in Fig. 1.

The demographic and clinical characteristics of the two groups were similar at baseline. More men (57.6\%) than women $(42.4 \%)$ were included in this trial $(p=0.525)$.

Average age of included patients was $61.8 \pm 10$ years in the OTG group and $59.4 \pm 12.5$ years in the MITG group $(p=0.298)$. Preoperative weight and BMI showed no difference, but a large group of patients suffered preoperative weight loss. All patients underwent a gastroscopy and staging CT, at the discretion of the local surgeon additional preoperative diagnostics were performed. A total of eight

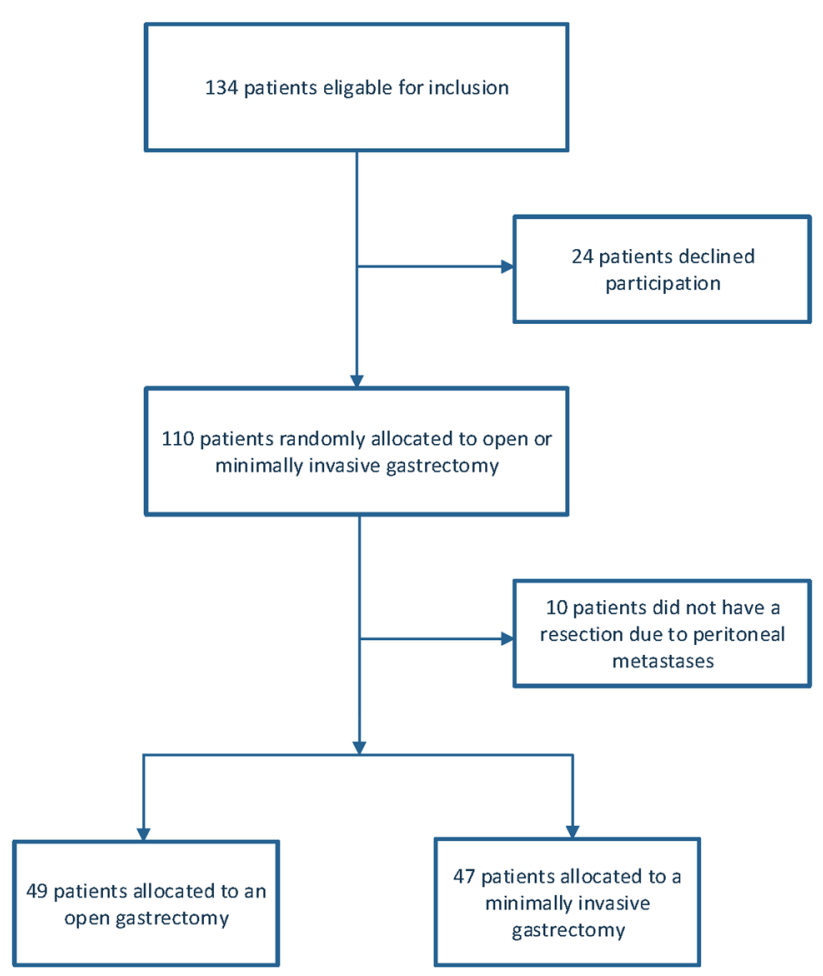

Fig. 1 Flowchart of the included patients 
patients underwent diagnostic laparoscopy to assess resectability prior to starting neoadjuvant chemotherapy.

In the OTG group 14 patients $(28.6 \%)$ had a proximal tumor, 25 patients $(51.0 \%)$ a middle tumor and ten patients (20.4\%) a distal tumor. In the MITG group 13 patients $(27.7 \%)$ had a proximal tumor, $25(53.2 \%)$ a middle tumor and nine (19.1\%) a distal tumor. There was no significant difference in distribution between both groups $(p=0.979)$. Baseline characteristics and preoperative workup are depicted in Table 1.

\section{Oncological outcomes}

Oncological safety of the procedure was measured as the number of retrieved lymph nodes and radicality of the procedure.

Pathological examination of the resected specimen showed that the numbers of retrieved lymph nodes and completeness of resection (i.e., resection margin (R0)) were similar between both groups. The mean number of resected lymph nodes was $43.4 \pm 17.3$ in OTG and $41.7 \pm 16.1$ in MITG $(p=0.612)$, mean difference 1.728 (95\% CI -5.02-8.47). For all lymph node stations data was collected as to whether the surgeon reported the station as resected. Next the pathologist confirmed resection for each tagged station if lymphoid tissue was found in that station. The number of harvested lymph nodes per station were also reported. No differences were observed in resected lymph node stations between both groups. A full D2 lymphadenectomy was performed in $49 \%$ of OTG versus $36.2 \%$ of MITG ( $p=0.223$ ). A splenectomy was performed in $12.2 \%$ OTG cases, versus $4.3 \%$ in MITG cases $(p=0.269)$.

There was no significant difference in radicality. Four patients had positive resection margins, one in the OTG and three in the MITG group. In $89.6 \%$ of all patient's histological report showed adenocarcinoma with $51.0 \%$ intestinal type in the OTG group versus $34.0 \%$ in the MITG group and $38.8 \%$ diffuse type in the OTG group versus $55.3 \%$ in the MITG group, this was not significantly different.

No significant differences were observed for textbook outcome. A textbook outcome was reached in $77,6 \%$ of patients in the OTG group versus $66 \%$ in the MITG group $(p=0.258)$.

An overview of all oncological results is depicted in Tables 3 and 4.

Regression techniques determined no confounding or effect modification by sex, age, BMI, T-stage and N-stage for these outcomes.

\section{Operative outcomes}

Although duration of surgery was significantly longer in the MITG group, with a median length of 244 min (IQR
198-293 $\mathrm{min}$ ) in comparison to $200 \mathrm{~min}$ (IQR 164-245 $\mathrm{min}$ ) in the OTG group $(p=0.005)$, blood loss was similar in both groups, with a median of $200 \mathrm{ml}$ in the OTG and $171 \mathrm{ml}$ in the MITG group $(p=0.454)$. In three patients the minimally invasive procedure was converted to an open procedure. Reasons for conversions were technical difficulty due to tumor infiltration, adhesions and perioperative hemodynamic instability.

In nine patients perioperative bleeding was reported, five in the MITG and four in the OTG group and one hospital reported an iatrogenic perforation of the colon which was directly repaired.

Operative details are depicted in Table 2.

\section{Postoperative outcomes}

Regarding postoperative recovery, outcomes were similar in both groups. Mean hospital stay was 8 days (IQR 7-11 days) in both groups if no complications occurred $(p=0.338)$. There was no significant difference in postoperative complications between OTG and MITG, with a total of $42.9 \%$ in OTG and $34.0 \%$ in MITG $(p=0.408)$. No differences were observed between OTG and MITG for major complications, being Clavien-Dindo grade 3 or higher $(p=0.377)$. Closer assessment of complications like postoperative bleeding, abscesses, anastomotic leakages (five in the OTG group, $10.2 \%$ and four in the MITG, 8.5\%), and wound dehiscence, one in every group, were not statistically different between OTG and MITG. Both circular stapled (54,2\%) and linear stapled $(45,8 \%)$ techniques were applied equally in OTG and MITG for the formation of the esophagojejunostomy $(p=0.546)$. No differences were observed in anastomotic leak rates between the two techniques $(p=0.501)$. In the OTG group two patients died after anastomotic leakage due to multi organ failure and sepsis, two other patients had a reoperation, and one was treated with antibiotics only. In the MITG group one patient had a reoperation, one had percutaneous drainage and two were treated with antibiotics. Median Comprehensive Complication Index was not significantly different between both groups with a mean of 20.9 (IQR 20.9-31.56) in the OTG and 26.22 (IQR 20.9-33.7) in the MITG group $(p=0.242)$.

The time to first intake was 5 days in both groups, as was the time to first passage of stool. Postoperative results are depicted in Table 5.

No differences were seen in the 1-year survival between the OTG and MITG group. In the OTG group $90.4 \%$ and in the MITG group $85.5 \%$ was alive 1 year after surgery $(p=0.701)$. One-year survival curves are depicted in Fig. 2 . 
Table 3 Pathology

\begin{tabular}{|c|c|c|c|c|c|}
\hline \multicolumn{6}{|l|}{ Pathology results } \\
\hline & Open $(n=49)$ & & MI $(n=47)$ & & $p$-value \\
\hline \multicolumn{6}{|l|}{ Pathology type } \\
\hline Intestinal type adenocarcinoma & 25 & $51.0 \%$ & 16 & $34.0 \%$ & $0.366^{*}$ \\
\hline Diffuse type adenocarcinoma & 19 & $38.8 \%$ & 26 & $55.3 \%$ & \\
\hline Carcinoid & 1 & $2.0 \%$ & 1 & $2.1 \%$ & \\
\hline Signet cell carcinoma & 3 & $6.1 \%$ & 1 & $2.1 \%$ & \\
\hline Other & 1 & $2.0 \%$ & 3 & $6.4 \%$ & \\
\hline \multicolumn{6}{|l|}{ ypT-stage } \\
\hline T0 (complete regression) & 4 & $8.2 \%$ & 3 & $6.4 \%$ & \\
\hline Tis & 2 & $4.1 \%$ & 1 & $2.1 \%$ & \\
\hline $\mathrm{T} 1$ & 8 & $16.3 \%$ & 6 & $12.8 \%$ & \\
\hline $\mathrm{T} 2$ & 4 & $8.2 \%$ & 5 & $10.6 \%$ & \\
\hline $\mathrm{T} 3$ & 19 & $38.8 \%$ & 17 & $36.2 \%$ & \\
\hline $\mathrm{T} 4$ & 12 & $24.5 \%$ & 15 & $31.9 \%$ & \\
\hline \multicolumn{6}{|l|}{ ypN-stage } \\
\hline No & 23 & $46.9 \%$ & 20 & $42.6 \%$ & \\
\hline N1 & 13 & $26.5 \%$ & 7 & $14.9 \%$ & \\
\hline $\mathrm{N} 2$ & 8 & $16.3 \%$ & 8 & $17.0 \%$ & \\
\hline N3 & 5 & $10.2 \%$ & 12 & $25.5 \%$ & \\
\hline \multicolumn{6}{|l|}{ Radical resection } \\
\hline Proximal margin & 0 & & 3 & $5 \%$ & 0.242 \\
\hline Distal margin & 1 & $2.2 \%$ & 0 & & 0.495 \\
\hline \multicolumn{6}{|l|}{ Lymph nodes } \\
\hline Total LN & 43.4 & \pm 17.3 & 41.7 & \pm 16.1 & 0.612 \\
\hline Micrometastasis & 3.2 & & 4.4 & & 0.514 \\
\hline Macrometastasis & 1.4 & & 2.1 & & 0.332 \\
\hline \multicolumn{6}{|l|}{ Station 1} \\
\hline Surgeon stated as resected & 49 & $100 \%$ & 47 & $100 \%$ & NA \\
\hline $\mathrm{LN}$ in station $(\mathrm{Y} / \mathrm{N})$ & 44 & $89.8 \%$ & 45 & $95.7 \%$ & 0.549 \\
\hline Average LN yield & 3 & $(2-6)$ & 3 & $(2-6)$ & 0.934 \\
\hline \multicolumn{6}{|l|}{ Station 2} \\
\hline Surgeon stated as resected & 49 & $100 \%$ & 47 & $100 \%$ & NA \\
\hline $\mathrm{LN}$ in station $(\mathrm{Y} / \mathrm{N})$ & 44 & $89.8 \%$ & 44 & $93.6 \%$ & 0.610 \\
\hline Average LN yield & 2 & $(1-4)$ & 2 & $(1-3)$ & 0.181 \\
\hline \multicolumn{6}{|l|}{ Station 3} \\
\hline Surgeon stated as resected & 49 & $100 \%$ & 47 & $100 \%$ & \\
\hline $\mathrm{LN}$ in station $(\mathrm{Y} / \mathrm{N})$ & 45 & $91.8 \%$ & 47 & $100.0 \%$ & 0.387 \\
\hline Average LN yield & 5 & $(3-15)$ & 3 & $(2-8)$ & 0.019 \\
\hline \multicolumn{6}{|l|}{ Station 4} \\
\hline Surgeon stated as resected & 49 & $100 \%$ & 47 & $100 \%$ & NA \\
\hline $\mathrm{LN}$ in station $(\mathrm{Y} / \mathrm{N})$ & 46 & $93.9 \%$ & 47 & $100.0 \%$ & 0.368 \\
\hline Average LN yield & 6 & $(2-11)$ & 5 & $(2-12)$ & 0.770 \\
\hline \multicolumn{6}{|l|}{ Station 5} \\
\hline Surgeon stated as resected & 48 & $98.0 \%$ & 45 & $95.7 \%$ & 0.613 \\
\hline $\mathrm{LN}$ in station $(\mathrm{Y} / \mathrm{N})$ & 45 & $93.8 \%$ & 44 & $97.8 \%$ & 0.549 \\
\hline Average LN yield & 1 & $(0-3)$ & 1 & $(0-3)$ & 0.381 \\
\hline \multicolumn{6}{|l|}{ Station 6} \\
\hline Surgeon stated as resected & 49 & $100 \%$ & 47 & $100 \%$ & NA \\
\hline $\mathrm{LN}$ in station $(\mathrm{Y} / \mathrm{N})$ & 45 & $91.8 \%$ & 46 & $97.9 \%$ & 0.513 \\
\hline Average LN yield & 4 & $(2-9)$ & 4 & $(2-7)$ & 0.487 \\
\hline
\end{tabular}


Table 3 (continued)

\begin{tabular}{|c|c|c|c|c|c|}
\hline \multicolumn{6}{|l|}{ Pathology results } \\
\hline & Open $(n=49)$ & & MI $(n=47)$ & & $p$-value \\
\hline \multicolumn{6}{|l|}{ Station 7} \\
\hline Surgeon stated as resected & 48 & $98.0 \%$ & 46 & $97.9 \%$ & 0.999 \\
\hline $\mathrm{LN}$ in station $(\mathrm{Y} / \mathrm{N})$ & 44 & $91.7 \%$ & 44 & $95.7 \%$ & 0.610 \\
\hline Average LN yield & 3.5 & $(2-7)$ & 3.5 & $(1-6.5)$ & 0.580 \\
\hline \multicolumn{6}{|l|}{ Station 8} \\
\hline Surgeon stated as resected & 48 & $98.0 \%$ & 45 & $95.7 \%$ & 0.613 \\
\hline $\mathrm{LN}$ in station $(\mathrm{Y} / \mathrm{N})$ & 46 & $95.8 \%$ & 42 & $93.3 \%$ & 0.147 \\
\hline \multicolumn{6}{|l|}{ Average LN yield } \\
\hline \multicolumn{6}{|l|}{ Station 9} \\
\hline Surgeon stated as resected & 49 & $100 \%$ & 44 & $93.6 \%$ & 0.113 \\
\hline $\mathrm{LN}$ in station $(\mathrm{Y} / \mathrm{N})$ & 39 & $79.6 \%$ & 40 & $90.9 \%$ & 0.589 \\
\hline Average LN yield & 2 & $(1-4)$ & 4 & $(1-6)$ & 0.125 \\
\hline \multicolumn{6}{|l|}{ Station 10} \\
\hline Surgeon stated as resected & 27 & $57.4 \%$ & 23 & $48.9 \%$ & 0.683 \\
\hline $\mathrm{LN}$ in station $(\mathrm{Y} / \mathrm{N})$ & 20 & $74.1 \%$ & 21 & $91.3 \%$ & 0.597 \\
\hline Average LN yield & 1.5 & $(0-4)$ & 1 & $(0-4)$ & 0.989 \\
\hline \multicolumn{6}{|l|}{ Station 11} \\
\hline Surgeon stated as resected & 42 & $89.4 \%$ & 36 & $76.6 \%$ & 0.302 \\
\hline $\mathrm{LN}$ in station $(\mathrm{Y} / \mathrm{N})$ & 35 & $83.3 \%$ & 30 & $83.3 \%$ & 0.327 \\
\hline Average LN yield & 2 & $(0-3)$ & 1 & $(0-3)$ & 0.796 \\
\hline \multicolumn{6}{|l|}{ Station 12} \\
\hline Surgeon stated as resected & 39 & $83.0 \%$ & 35 & $74.5 \%$ & 0.631 \\
\hline $\mathrm{LN}$ in station $(\mathrm{Y} / \mathrm{N})$ & 28 & $71.8 \%$ & 31 & $88.6 \%$ & 0.52 \\
\hline Average LN yield & 1 & $(0-2)$ & 2 & $(0-4)$ & 0.096 \\
\hline \multicolumn{6}{|l|}{ Station 13} \\
\hline Surgeon stated as resected & 1 & $2.1 \%$ & 0 & & 0.999 \\
\hline $\mathrm{LN}$ in station $(\mathrm{Y} / \mathrm{N})$ & 0 & & 0 & & \\
\hline Average LN yield & - & & - & & \\
\hline
\end{tabular}

* Additional testing within groups with Bonferoni correction showed no differences between groups

** Additional testing within groups revealed there were significantly more N3 patients in the minimally invasive group

\section{Discussion}

In this trial, MITG and OTG after neoadjuvant therapy resulted in similar oncological outcomes; measured by number of retrieved lymph nodes, lymph node stations resected, radicality of the resection and 1-year survival. This is the first European randomized trial evaluating oncological outcomes in MITG and OTG. The short-term results of this trial indicate that MITG is non-inferior to OTG regarding oncological and surgical safety.

In gastric cancer, a radical resection and adequate lymph node dissection has shown to be an important prognostic factor and is a marker for the quality of surgery, and thereby quality of care. According to the ESMO guidelines an adequate lymph node dissection comprises of a D2 dissection with a minimum of 15 lymph nodes [3]. This number represents the minimum for an adequate staging but also for adequate resection. In this trial the mean number of resected lymph nodes was $43.4 \pm 17.3$ in OTG and $41.7 \pm 16.1$ in MITG group $(p=0.612)$ and within the range of the predetermined non-inferiority margin. Looking more closely at the resected lymph node stations for an adequate D2 lymphadenectomy no differences were observed between the two groups. It should be noted that the successful D2 lymphadenectomy rate was rather low, which was mainly due to inadequate dissection of station 10. Standard dissection of station 10 is no longer recommended in the latest JGCA guidelines unless the tumor invades the greater curvature [22].

Due to the low incidence of gastric cancer in the West compared to Asia, no screening program exists, and patients often present with advanced disease stages [2]. In this study over $60 \%$ in both MITG and OTG presented with cT3-4 stages. Due to the majority of patients presenting with more 
Table 4 Oncological results

\begin{tabular}{|c|c|c|c|c|c|c|}
\hline \multicolumn{7}{|l|}{ Oncological results } \\
\hline & & \multicolumn{2}{|c|}{ Open $(n=49)$} & \multicolumn{2}{|c|}{ MI $(n=47)$} & \multirow[t]{2}{*}{$p$ value } \\
\hline \multicolumn{6}{|l|}{ Lymph nodes } & \\
\hline Total $($ mean $\pm \mathrm{SD})$ & & 44.3 & \pm 16.7 & 40.7 & \pm 16.3 & 0.209 \\
\hline Micrometastasis & & 3.2 & & 4.4 & & 0.514 \\
\hline Macrometastasis & & 1.4 & & 2.1 & & 0.332 \\
\hline \multicolumn{7}{|l|}{ Radicality } \\
\hline $\mathrm{R} 0$ resection & & 48 & $98.0 \%$ & 44 & $93.6 \%$ & 0.617 \\
\hline Positive proximal margin & & 0 & & 3 & $5.0 \%$ & 0.242 \\
\hline Positive distal margin & & 1 & $2.2 \%$ & 0 & & 0.495 \\
\hline Mandard classification $^{\mathrm{a}}$ & & & & & & $0.934 *$ \\
\hline 1 & Complete regression (= fibrosis without detectable tissue of tumor) & 6 & $14.0 \%$ & 5 & $11.4 \%$ & \\
\hline 2 & Fibrosis with scattered tumor cells & 7 & $16.3 \%$ & 5 & $11.4 \%$ & \\
\hline 3 & Fibrosis and tumor cells with preponderance of fibrosis & 7 & $16.3 \%$ & 9 & $20.5 \%$ & \\
\hline 4 & Fibrosis and tumor cells with preponderance of tumor cells & 12 & $27.9 \%$ & 12 & $27.3 \%$ & \\
\hline 5 & Tissue of tumor without changes of regression & 11 & $25.6 \%$ & 13 & $29.5 \%$ & \\
\hline
\end{tabular}

* Additional testing within groups with Bonferroni correction showed no differences between groups

${ }^{a}$ Data missing of 9 patients

advanced stages of disease, neoadjuvant therapy is more common in the West compared to Asia.

Alongside a higher rate of comorbidities (i.e., cardiovascular, endocrine, pulmonal diseases, and obesity. Measured overall with the ASA classification) was observed. Although average BMI was higher, patients often suffered preoperative weight loss, indicating a poor nutritional status.

In Northern Europe the incidence of gastric cancer has stabilized. Active eradication of Helicobactor Pylori, and diet changes leading up to ingestion of more fresh products (and less preservatives) have led to a decreased incidence in distal gastric cancers, whereas the incidence for proximal and middle gastric cancers seems stable [1]. Indicating the need for optimal surgical strategies for total gastrectomy in these patients.

Postoperative recovery and postoperative morbidity and mortality was similar in both groups. Previous studies on minimally invasive gastrectomy focused mainly on distal gastrectomy, such as the South Korean KLASS trials [23, 24]. These trials showed that minimally invasive distal gastrectomy is oncologically safe with improved postoperative outcomes such as a lower complication rate, faster recovery and less pain compared to open surgery. The short-term advantages found in these trials with distal gastrectomy have not be found in our study, where there are no differences in short-term outcomes between both approaches.
Concerning total gastrectomy, some meta-analyses have been performed showing that MITG had longer operation times, less operative blood loss, similar rates in lymph node yield with the same completeness of the resection, faster postoperative recovery, less postoperative complications with shorter length of hospital stay and similar mortality rates. Both meta-analyses have emphasized the need for a prospective randomized trial comparing MITG and OTG $[25,26]$. In a single arm confirmatory trial the safety of LATG was further emphasized [27].

The outcomes regarding postoperative recovery from our trial might be explained by the multi-disciplinary approach to perioperative care. For example, clinical care pathways ensure better postoperative pain management with dedicated pain teams. Alongside, standardized postoperative patient care protocols ensure optimal perioperative care and allows early diagnosis and treatment of complications.

The South Korean KLASS 03 feasibility study on MITG on patients with stage I proximal gastric cancer has found postoperative morbidity rates of $20.6 \%$ with grade III or higher of $9.4 \%$ and a mortality of $0.6 \%$ [28].

The outcome of our trial reflects the daily practice in the West with less incidence of gastric cancer, where centralization in high volume centers, implementation of minimally invasive gastrectomy, and adoption of new neoadjuvant chemotherapy are important items. 
Table 5 Postoperative results

Postoperative results

\begin{tabular}{|c|c|c|c|c|c|}
\hline \multirow[b]{2}{*}{ Complications } & \multicolumn{2}{|c|}{$\begin{array}{l}\text { Open } \\
(n=49)\end{array}$} & \multicolumn{2}{|l|}{$\begin{array}{l}\text { MI } \\
(n=47)\end{array}$} & \multirow{2}{*}{$\begin{array}{c}p \text {-value } \\
0.408\end{array}$} \\
\hline & 21 & $42.9 \%$ & 16 & $34.0 \%$ & \\
\hline \multicolumn{6}{|l|}{ Clavien-Dindo } \\
\hline CD 1 & 3 & $6.1 \%$ & 1 & $2.1 \%$ & \multirow[t]{6}{*}{$0.377 *$} \\
\hline $\mathrm{CD} 2$ & 12 & $24.5 \%$ & 7 & $14.9 \%$ & \\
\hline $\mathrm{CD} 3 \mathrm{~A}$ & 1 & $2.0 \%$ & 3 & $6.4 \%$ & \\
\hline $\mathrm{CD} 3 \mathrm{~B}$ & 2 & $4.1 \%$ & 2 & $4.3 \%$ & \\
\hline $\mathrm{CD} 4$ & 1 & $2.0 \%$ & 3 & $6.4 \%$ & \\
\hline CD 5 & 2 & $4.1 \%$ & 0 & - & \\
\hline Anastomotic Leak & 5 & $10.2 \%$ & 4 & $8.5 \%$ & 0.527 \\
\hline Abscess & 1 & $2.0 \%$ & 1 & $2.1 \%$ & 0.742 \\
\hline Perforation & 1 & $2.0 \%$ & 1 & $2.1 \%$ & 0.742 \\
\hline Wound infection & 1 & $2.0 \%$ & 1 & $2.1 \%$ & 0.742 \\
\hline Fascial dehiscence & 0 & & 0 & & \\
\hline Pneumonia & 3 & $6.1 \%$ & 4 & $8.5 \%$ & 0.712 \\
\hline Urinary tract infection & 1 & $2.0 \%$ & 0 & & 0.510 \\
\hline Bleeding & 3 & $6.1 \%$ & 0 & & 0.494 \\
\hline Cardial & 1 & $2.0 \%$ & 1 & $2.1 \%$ & 0.742 \\
\hline Thrombo-embolic & 2 & $4.1 \%$ & 1 & $2.3 \%$ & 0.999 \\
\hline Other & 8 & & 11 & & \\
\hline \multicolumn{5}{|c|}{ Technique for anastomosis** } & \multirow[t]{5}{*}{0.546} \\
\hline Circular stapled & 25 & $49 \%$ & 27 & $57 \%$ & \\
\hline Anastomotic leak & 3 & & 3 & & \\
\hline Linear stapled with V-loc & 24 & $51 \%$ & 20 & $43 \%$ & \\
\hline Anastomotic leak & 2 & & 1 & & \\
\hline \multicolumn{6}{|c|}{ Postoperative recovery (days) } \\
\hline \multicolumn{6}{|l|}{ Hospital stay } \\
\hline Uncomplicated & 8 & $(7-11)$ & 8 & $(7-9)$ & 0.338 \\
\hline Complicated & 11 & $(9-15)$ & 10 & $(8-17)$ & 0.452 \\
\hline First intake & 4 & $(3-6)$ & 4 & $(3-6)$ & 0.641 \\
\hline First stool & 5 & $(4-6)$ & 5 & $(4-6)$ & 0.707 \\
\hline
\end{tabular}

* Additional testing within groups with Bonferroni correction showed no differences between group

${ }^{a}$ No differences were observed in anastomotic leak rates between the two techniques $(p=0.501)$
Because strict exclusion criteria were met, accrual of patients was slower than expected.

This was attributed to several factors. As reported earlier a large group of patients were not deemed fit for neoadjuvant chemotherapy and, therefore, not included in this trial. In addition, only patients receiving total gastrectomy could be included to maintain a homogenous study population. This is an important shortcoming in the trial, as the sample size was re-adjusted to a lower sample size to ensure adequate completion of the trial.

The discrepancy between postoperative and preoperative staging was remarkable, with many patients being under staged preoperatively. Ten patients were excluded in the trial due to perioperative diagnosis of peritoneal metastases. Despite thorough preoperative workup in patients with gastric cancer, adequate staging remains a challenge [29].

This trial is the first trial to compare MITG and OTG in a Western population, taking into account neoadjuvant treatment. Long-term outcomes concerning survival and disease-free survival will be collected to evaluate long-term results. However, it should be noted that this study was not powered on disease free and overall survival. In addition, future research should also assess quality of life in patients with advanced gastric cancer.

\section{Conclusion}

The results of this trial provide evidence of non-inferiority regarding quality of the oncological resection in MITG compared to OTG in the treatment of advanced gastric cancer. This indicates that MITG is safe from an oncological point of view. Postoperative complications and recovery are similar in OTG and MITG. Awaiting the results of quality of life and 3-year overall survival, currently the type of approach is at the discretion of the surgical team and may depend on patient factors and patient's choice. 
Fig. 2 1-year survival

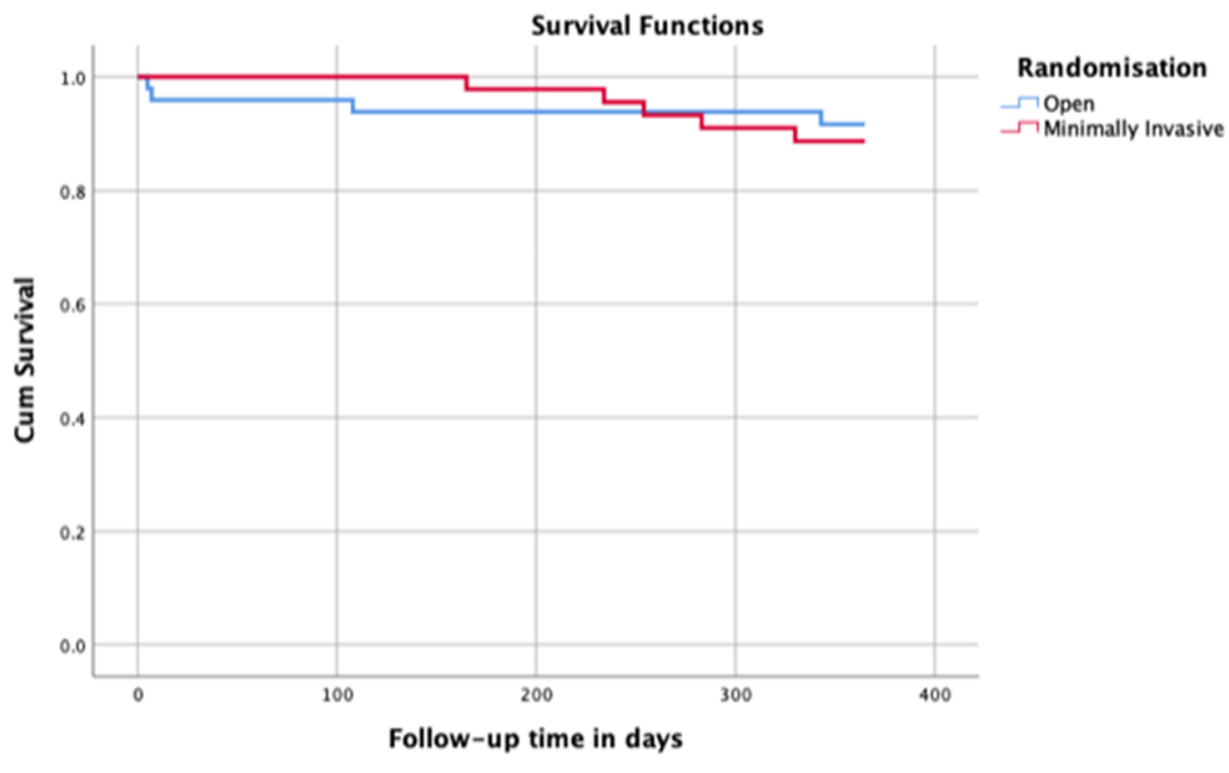

\begin{tabular}{llllll}
\multicolumn{2}{c}{ Numbers at risk } & & & & \\
Time in days & 0 & 100 & 200 & 300 & 365 \\
Open & 45 & 41 & 32 & 30 & 26 \\
MI & 41 & 40 & 34 & 28 & 26
\end{tabular}

\section{Compliance with ethical standards}

Disclosure of potential conflict of interest The STOMACH trial received funding by Fonds NutsOhra (FNO). This was given to the department of gastro-intestinal surgery at Amsterdam University Medical Center, location VU university. All other authors declare no conflict of interest.

Human rights statement and informed consent All procedures followed were in accordance with the ethical standards of the responsible committee on human experimentation (institutional and national) and with the Helsinki Declaration of 1964 and later versions. Informed consent to be included in the study was obtained from all patients.

Open Access This article is licensed under a Creative Commons Attribution 4.0 International License, which permits use, sharing, adaptation, distribution and reproduction in any medium or format, as long as you give appropriate credit to the original author(s) and the source, provide a link to the Creative Commons licence, and indicate if changes were made. The images or other third party material in this article are included in the article's Creative Commons licence, unless indicated otherwise in a credit line to the material. If material is not included in the article's Creative Commons licence and your intended use is not permitted by statutory regulation or exceeds the permitted use, you will need to obtain permission directly from the copyright holder. To view a copy of this licence, visit http://creativecommons.org/licenses/by/4.0/.

\section{References}

1. Rawla P, Barsouk A. Epidemiology of gastric cancer: global trends, risk factors and prevention. Prz Gastroenterol. 2019;14(1):26-38.
2. Rausei S, Boni L, Rovera F, Dionigi G. Locally advanced gastric cancer: a new definition to standardise. J Clin Pathol. 2013;66(2):164-5.

3. Smyth EC, Verheij M, Allum W, Cunningham D, Cervantes A, Arnold D, et al. Gastric cancer: ESMO clinical practice guidelines for diagnosis, treatment and follow-up. Ann Oncol. 2016;27(suppl 5): $\mathrm{v} 38-49$.

4. Kim W, Kim HH, Han SU, Kim MC, Hyung WJ, Ryu SW, et al. Decreased morbidity of laparoscopic distal gastrectomy compared with open distal gastrectomy for stage i gastric cancer: shortterm outcomes from a multicenter randomized controlled trial (KLASS-01). Ann Surg. 2016;263(1):28-35.

5. Beyer K, Baukloh AK, Kamphues C, Seeliger H, Heidecke $\mathrm{CD}$, Kreis ME, et al. Laparoscopic versus open gastrectomy for locally advanced gastric cancer: a systematic review and metaanalysis of randomized controlled studies. World J Surg Oncol. 2019; 17(1):68.

6. Chen XZ, Wang SY, Wang YS, Jiang ZH, Zhang WH, Liu K, et al. Comparisons of short-term and survival outcomes of laparoscopyassisted versus open total gastrectomy for gastric cancer patients. Oncotarget. 2017;8(32):52366-80.

7. Ferlay J, Soerjomataram I, Dikshit R, Eser S, Mathers C, Rebelo $\mathrm{M}$, et al. Cancer incidence and mortality worldwide: sources, methods and major patterns in GLOBOCAN 2012. Int J Cancer. 2015;136(5):E359-86.

8. Leja M, You W, Camargo MC, Saito H. Implementation of gastric cancer screening - the global experience. Best Pract Res Clin Gastroenterol. 2014;28(6):1093-106.

9. van der Wielen N, Straatman J, Cuesta MA, Daams F, van der Peet DL. Short-term outcomes in minimally invasive versus open gastrectomy: the differences between East and West. A systematic review of the literature. Gastric Cancer. 2018;21(1):19-30.

10. Cunningham D, Allum WH, Stenning SP, Thompson JN, Van de Velde CJ, Nicolson M, et al. Perioperative chemotherapy versus surgery alone for resectable gastroesophageal cancer. N Engl J Med. 2006;355(1):11-20. 
11. Al-Batran SE, Homann N, Pauligk C, Goetze TO, Meiler J, Kasper $\mathrm{S}$, et al. Perioperative chemotherapy with fluorouracil plus leucovorin, oxaliplatin, and docetaxel versus fluorouracil or capecitabine plus cisplatin and epirubicin for locally advanced, resectable gastric or gastro-oesophageal junction adenocarcinoma (FLOT4): a randomised, phase 2/3 trial. Lancet. 2019;393(10184):1948-57.

12. Huscher CG, Mingoli A, Sgarzini G, Sansonetti A, Di Paola M, Recher A, et al. Laparoscopic versus open subtotal gastrectomy for distal gastric cancer: five-year results of a randomized prospective trial. Ann Surg. 2005;241(2):232-7.

13. Straatman J, van der Wielen N, Cuesta MA, Gisbertz SS, Hartemink KJ, Alonso Poza A, et al. Surgical techniques, open versus minimally invasive gastrectomy after chemotherapy (STOMACH trial): study protocol for a randomized controlled trial. Trials. 2015; $16: 123$

14. Japanese Gastric Cancer A. Japanese gastric cancer treatment guidelines 2014 (ver. 4). Gastric Cancer. 2017;20(1):1-19.

15. Thies S, Langer R. Tumor regression grading of gastrointestinal carcinomas after neoadjuvant treatment. Front Oncol. 2013;3:262.

16. Clavien PA, Barkun J, de Oliveira ML, Vauthey JN, Dindo $\mathrm{D}$, Schulick RD, et al. The Clavien-Dindo classification of surgical complications: five-year experience. Ann Surg. 2009;250(2):187-96.

17. Slankamenac K, Graf R, Barkun J, Puhan MA, Clavien PA. The comprehensive complication index: a novel continuous scale to measure surgical morbidity. Ann Surg. 2013;258(1):1-7.

18. Busweiler LA, Schouwenburg MG, van Berge Henegouwen MI, Kolfschoten NE, de Jong PC, Rozema T, et al. Textbook outcome as a composite measure in oesophagogastric cancer surgery. Br J Surg. 2017;104(6):742-50.

19. Julious SA. Sample sizes for clinical trials with normal data. Stat Med. 2004;23(12):1921-86.

20. Audit DUGC. Annual Report 2013. 2014.

21. Dikken JL, van Grieken NC, Krijnen P, Gonen M, Tang LH, Cats A, et al. Preoperative chemotherapy does not influence the number of evaluable lymph nodes in resected gastric cancer. Eur J Surg Oncol. 2012;38(4):319-25.

22. Japanese Gastric Cancer A. Japanese gastric cancer treatment guidelines 2018 (5th edition). Gastric Cancer. 2020. Epub 2020/02/16.
23. Kim HH, Han SU, Kim MC, Kim W, Lee HJ, Ryu SW, et al. Effect of laparoscopic distal gastrectomy vs open distal gastrectomy on long-term survival among patients with stage I gastric cancer: the KLASS-01 randomized clinical trial. JAMA Oncol. 2019;5(4):506-13.

24. Lee HJ, Hyung WJ, Yang HK, Han SU, Park YK, An JY, et al. Short-term outcomes of a multicenter randomized controlled trial comparing laparoscopic distal gastrectomy with D2 lymphadenectomy to open distal gastrectomy for locally advanced gastric cancer (KLASS-02-RCT). Ann Surg. 2019;270(6):983-91.

25. Haverkamp L, Weijs TJ, van der Sluis PC, van der Tweel I, Ruurda JP, van Hillegersberg R. Laparoscopic total gastrectomy versus open total gastrectomy for cancer: a systematic review and metaanalysis. Surg Endosc. 2013;27(5):1509-20.

26. Straatman J, van der Wielen N, Cuesta MA, de Lange-de Klerk ES, Jansma EP, van der Peet DL. Minimally invasive versus open total gastrectomy for gastric cancer: a systematic review and meta-analysis of short-term outcomes and completeness of resection: surgical techniques in gastric cancer. World J Surg. 2016;40(1):148-57.

27. Katai H, Mizusawa J, Katayama H, Kunisaki C, Sakuramoto $\mathrm{S}$, Inaki N, et al. Single-arm confirmatory trial of laparoscopyassisted total or proximal gastrectomy with nodal dissection for clinical stage I gastric cancer: Japan Clinical Oncology Group study JCOG1401. Gastric Cancer. 2019;22(5):999-1008.

28. Hyung WJ, Yang HK, Han SU, Lee YJ, Park JM, Kim JJ, et al. A feasibility study of laparoscopic total gastrectomy for clinical stage I gastric cancer: a prospective multi-center phase II clinical trial, KLASS 03. Gastric Cancer. 2019;22(1):214-22.

29. Mocellin S, Pasquali S. Diagnostic accuracy of endoscopic ultrasonography (EUS) for the preoperative locoregional staging of primary gastric cancer. Cochrane Database Syst Rev. 2015;2:CD009944.

Publisher's Note Springer Nature remains neutral with regard to jurisdictional claims in published maps and institutional affiliations.

\title{
Affiliations
}

\author{
Nicole van der Wielen ${ }^{1}\left[\right.$. Jennifer Straatman ${ }^{1,2} \cdot$ Freek Daams $^{1} \cdot$ Riccardo Rosati $^{3} \cdot$ Paolo Parise $^{3}$. \\ Jürgen Weitz ${ }^{4}$. Christoph Reissfelder ${ }^{5}$. Ismael Diez del Val ${ }^{6}$. Carlos Loureiro ${ }^{6}$. Purificación Parada-González ${ }^{7}$. \\ Elena Pintos-Martínez ${ }^{7}$. Francisco Mateo Vallejo ${ }^{8} \cdot$ Carlos Medina Achirica $^{8}$. Andrés Sánchez-Pernaute ${ }^{9}$. \\ Adriana Ruano Campos ${ }^{9}$. Luigi Bonavina ${ }^{10}$. Emanuele L. G. Asti ${ }^{10}$. Alfredo Alonso Poza ${ }^{11}$. Carlos Gilsanz ${ }^{11}$. \\ Magnus Nilsson ${ }^{12}$. Mats Lindblad ${ }^{12}$. Suzanne S. Gisbertz ${ }^{13}$. Mark I. van Berge Henegouwen ${ }^{13}$. \\ Uberto Fumagalli Romario ${ }^{14} \cdot$ Stefano De Pascale ${ }^{14} \cdot$ Khurshid Akhtar $^{15} \cdot$ H. Jaap Bonjer ${ }^{1} \cdot$ Miguel A. Cuesta $^{1}$. \\ Donald L. van der Peet ${ }^{1}$
}

1 Department of Gastro-Intestinal Surgery, Amsterdam University Medical Center, Location VU University, De Boelelaan 1117, ZH 7F020, 1081 HV Amsterdam, The Netherlands

2 Department of Clinical Epidemiology, Amsterdam University Medical Center, Amsterdam, The Netherlands

3 Department of Surgery, San Raffaele Hospital, Milan, Italy
4 Department of Visceral-, Thoracic and Vascular Surgery, University Hospital Carl Gustav Carus, Dresden, Germany

5 Department of Surgery, Universitätsmedizin Mannheim, Medical Faculty Mannheim, Heidelberg University, Heidelberg, Germany

6 Department of Surgery, Hospital Universitario de Basurto, Bilbao, Spain 
7 Department of Surgery, Hospital Clínico Universitario de Santiago de Compostela, Santiago de Compostela, Spain

8 Department of Surgery, Hospital de Jerez, Jerez de la Frontera, Spain

9 Department of Surgery, Hospital Clínico San Carlos, Madrid, Spain

10 Department of Surgery, IRCCS Policlinico San Donato, Milan, Italy

11 Department of Surgery, Hospital del Sureste, Madrid, Spain
12 Department of Surgery, Karolinska University Hospital, Stockholm, Sweden

13 Department of Gastro-intestinal Surgery, Amsterdam University Medical Center Location AMC, Amsterdam, The Netherlands

14 Department of Surgery, ASST Spedali Civili, Brescia, Italy

15 Department of Surgery, Salford Royal NHS Foundation Trust, Manchester, UK 